\title{
Improved SLIC Superpixel Segmentation Based on HSV Non-uniform Quantization
}

\author{
Dongping $\mathrm{Li}^{\mathrm{a}}$, Changliang Liu \\ North China Electric Power University, Baoding,071003, China \\ aemail:lidpncepu@163.com
}

Keywords: SLIC, superpixel, segmentation, HSV, quantization

\begin{abstract}
Simple linear iterative clustering (SLIC) method needs to artificially set the number of superpixel required for segmentation, which will easily lead to over-segmentation or under-segmentation, and that results can not meet the visual requirements. To improve the SLIC superpixel segmentation method, HSV non-uniform quantization, on the basis of color information,is proposed to get the picture feature vector for visual perception oriented, and then get the superpixel numbers of images needed. Experiments show that, over the number of superpixel obtained by this method, images can be partitioned into more desirably regions.
\end{abstract}

\section{Introduction}

How to effectively segment the target object from the image has been one of the classic problems in computer vision and image understanding, which determines the final discrimination result of the image quality analysis and pattern recognition. Image segmentation refers to separating the image into different regions for special meaning, disjointing these regions, and each specific region meet the conditions of consistency. The conventional image segmentation methods process targets on the basic pixel, does not take into account the spatial and organizational relationships between pixels.But along with the continuous development of technology, the size of the split image becomes larger and larger, resulting in low processing efficiency of the algorithm.The superpixel method[1],proposed by Rend et al.,uses the image over-segmentation scale ideological to generate a plurality of homogeneity of regions.It captures redundancy of images, which is more conducive to calculate the image feature, and reduces the complexity of the the subsequent images process. Therefore, methods based on superpixel segmentation, are widely used in image segmentation pretreatment process for its good segmentation boundary, efficient processing speed and lower computational complexity.

Currently, there are two main categories to produce superpixels: one bases on graph theory and the other bases on gradient ascent method. Graph-based theory segmentation method is a holistic approach to segmentation, the main idea of this method is the original image is mapped to a undirected weighted graph, each node corresponds to each pixel in the original graph, each edge of the graph corresponds to the relationship between the adjacent pixels, the edge weight corresponds to characteristics difference between adjacent pixels, then according to the threshold combined by the weight to segment image.Its representative algorithms are Normalized Cuts (N-Cuts)[2], Graph-Based (GS)[3], Superpixel Lattices (SL)[4]. Gradient-ascent-based segmentation is firstly obtained by a rough clustering, then uses the gradient ascent method to improve the result of the previous iteration until convergence. Its representative algorithms are Mean Shift[5],Quick Shift[6],Simple Linear Iterative Clustering(SLIC)[7]. Among them, SLIC is currently one of the best methods of superpixel segmentation algorithm for it uses similarity of luminance and distance feature to produce uniform size,regular shape and easy controlled super pixel block.And efficiency has been improved compared to other methods. 


\section{SLIC Segmentation Algorithm}

SLIC is a basic idea proposed by Adianta et al. It is simple and easy to implement. SLIC complete the local clustering of image pixels in a five dimensional space, the 5D is composed of CIELAB color space values $l, a, b$ and pixel position values $x, y$. At the same time, a new distance measure is proposed to control the shape, compactness and regularity of superpixels.It reflects a good application in color image. Experiments show high efficiency of SLIC algorithm, and it can produce high compactness and orderly rows of superpixels.

By calculating the similarity of pixels in color and proximity of spatial location ,SLIC algorithm clusters image to get superpixels. This is conducted in a five dimensional space $[l, a, b, x, y]$, where $[l, a, b]$ is the color vector in CIELAB space and $[x, y]$ is the space coordinate of pixels. In this five dimensional space, we can not simply use the Euclidean distance to measure the distance between pixels, so the clustering uses a normalized distance to pixel measurement. The normalized Euclidean distance can fuse the color similarity and the pixel position.

In an image with $N$ pixels, the number of superpixel is set to $K$, the size of each superpixel is $N / K$, the distance between the center of each superpixel is $S=\sqrt{N / K}$. In the algorithm, we choose the superpixel center as:

$$
C_{k}=\left[l_{k}, a_{k}, b_{k}, x_{k}, y_{k}\right]
$$

Because the spatial range of any superpixel is about $S^{2}$ (a superpixel's roughly region), the pixel in the rectangular area of the cluster center is the search range of this superpixel clustering process. In order to reduce the computation of the algorithm, each cluster center searches pixels only in its $2 S \times 2 S$ field. As a result of this narrowing search rather than global search, SLIC algorithm converges very fast in iterative process. The schematic diagram of SLIC clustering search is shown in Figure 1.

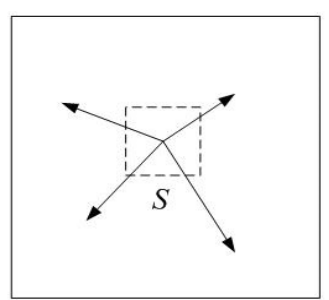

(a)

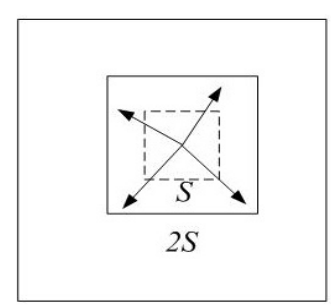

(b)

Figure 1. The schematic diagram of SLIC clustering search

In the similarity measure of pixels, the similarity degree between each pixel point and the nearest seed point is calculated, and the label of the most similar seed point is assigned to the pixel. The similarity between the pixels is as follows:

$$
\begin{aligned}
& d_{l a b}=\sqrt{\left(l_{k}-l_{i}\right)^{2}+\left(a_{k}-a_{i}\right)^{2}+\left(b_{k}-b_{i}\right)^{2}} \\
& d_{x y}=\sqrt{\left(x_{k}-x_{i}\right)^{2}+\left(y_{k}-y_{i}\right)^{2}} \\
& D_{s}=d_{\text {lab }}+\frac{m}{s} d_{x y}
\end{aligned}
$$

where, $D_{s}$ is the sum of the lab distance and the $x y$ plane distance normalized by the grid interval S.Compact factor $m$ is used to control the superpixel tightness, the value of $m$ is between [1,20], the default is 10 , the greater its value is, the more emphasis on spatial proximity, which makes the clustering more closely.

Given $K$ cluster centers, in order to avoid the clustering center just at the edge of the image, and at the same time reduce the possibility of the selected noise points, the algorithm will move cluster centers to a minimum gradient at $3 \times 3$ fields. The formula for calculating the image gradient is:

$$
G(x, y)=\|I(x+1, y)-I(x-1, y)\|^{2}+\|I(x, y+1)-I(x, y-1)\|^{2}
$$


where $I(x, y)$ is the $l a b$ vector corresponding to the pixel at position $(x, y)$, and $\|\cdot\|$ is the $L_{2}$ norm. This gives attention to both color and intensity information. Each image pixel contact with the nearest cluster center, when all the pixels are covered by the clustering region, by calculating the average of $l, a, b, x, y$ of all pixels in clustering region, a new center is generated. Repeat this process until the algorithm converges.

\section{Improved SLIC Segmentation Algorithm}

However, in the SLIC method above, it is necessary to set the number of supoerpixels in the image segmentation, which will cause a great impact on the segmentation results. Aiming at the problems existing in the SLIC superpixel segmentation, using color information to automatically get the number of superpixels is proposed. When the image to be processed is large, its color contains lots of information, if scan it entirety, it will take up too much unnecessary running time and storage space,so assumes that the image size is $M \times N$, randomly scan images of $M / e$ lines or $N / e$ columns, $e$ is the balance parameter to control the number of color information required. Experiments prove that when $e$ equals to 10, it can get satisfactory results.

In the field of image processing, color image analysis is generally in RGB mode, but the RGB color space is not intuitive , the RGB value is difficult to judge the color it represents, and there are great differences between RGB color space and human perception. HSV color space reflects the visual observation of the color of the human eye, and it's also conducive to image processing, so we convert RGB model into HSV model. $C_{\max }$ equals to $(R, G, B)$ in the maximum, $C_{\min }$ equals to $(R, G$, $B)$ in the minimum. Calculate the HSV space values $(H, S, V)$, which, $H \in[0,360], S, V \in[0,1]$, the formula for:

$$
\begin{aligned}
& H=\left\{\begin{array}{c}
(G-B) /\left(C_{\max }-C_{\min }\right), R=C_{\max } \\
2+(B-R) /\left(C_{\max }-C_{\min }\right), G=C_{\max } \\
4+(R-G) /\left(C_{\max }-C_{\min }\right), B=C_{\max }
\end{array}\right. \\
& S=\left(C_{\max }-C_{\min }\right) / C_{\max } \\
& V=C_{\max }
\end{aligned}
$$

Generally,True color of the color image is very large, and the dimension of the histogram vector will be too much. If we firstly quantify the HSV space appropriately, and then calculate the histogram, the calculation would be far less. According to human visual perception characteristics of color, we quantify $H, S, V$ in non-uniform model. $H$ is in the range of $[0,360] . S$ is in the range of $[0,1] . V$ is in the range of $[0,1]$.According to the human visual ability to distinguish different ranges of color and subjective color perception, the hue $H$ is quantized to sixteen levels, and saturation $S$ and luminance $V$ to four levels. Then, the three color components are combined into one dimensional feature vector $L$ :

$$
L=H Q_{s} Q_{v}+S Q_{v}+V=16 H+4 S+V
$$

Thus, $H, S$ and $V$ are distributed on one dimension vector. The range of $L$ is $[0,255]$. Calculate $L$ to get a 256 bin one-dimensional histogram.

Then count the number of times the same values $L$ appeare, and store it in a one-dimensional array $T$. Sort each value in $T$ from small to large, find their average ave, select the minimum value of $T$ which is larger than ave as the number of superpixels in the image.

In conclusion, the improved SLIC algorithm steps are as follows:

1.Random scan the image to obtain the M/e lines or N/e columns pixels' color information.

2.Change the scan pixels from RGB color information into HSV color information.

3.Quantify HSV in non-uniform model, and then combined the three color components into one dimension feature vector.

4. Determine the number of superpixels.

5. Given the initial cluster center $C_{k}=\left[l_{k}, a_{k}, b_{k}, x_{k}, y_{k}\right]^{T}$, the cluster center is generated through the sampling grid. 
6. Move cluster centers to the minimum gradient in $n \times n$ field.

7. For each cluster center $C_{k}$, according to the distance formula (2) to (4), search for similar pixels and mark in the $2 S \times 2 S$ field.

8. Calculate the new cluster center and research.

9. Until the $E$ is less than or equal to threshold ( $E$ is the difference between the new clustering center and original cluster center).

10. Enforce connectivity.

\section{Experiments}

Experiments use three different color images to verify the method above, the size of them are $481 \times 321$ (Image 1),816×616(Image 2),768×512(Image 3).

After transverse random scanning Image 1,calculate the pixel points to get the number of the same value in one-dimensional feature vector $L$, which is shown in Figure 2. Then get minimum superpixel value 138, which is larger than the average value,as the desired image superpixels.

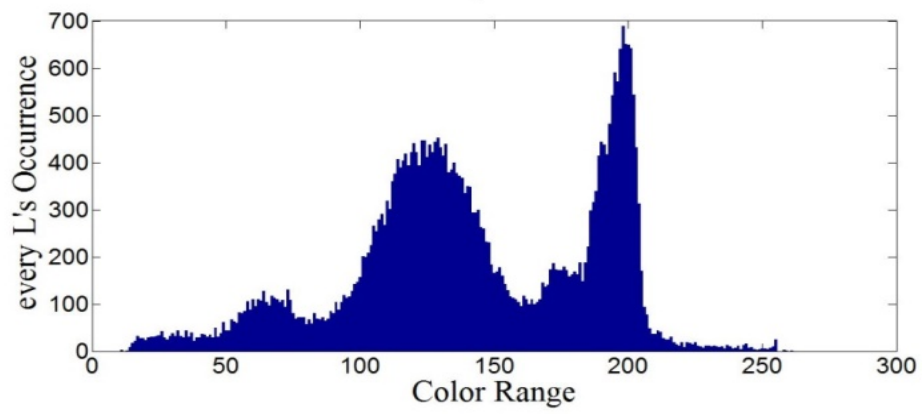

Figure 2 Value distribution of feature vector $L$

In Table 1, seven sets of values are shown, which are the number of superpixels of Image 1 , Image 2, Image 3 in horizontal and vertical random scans. As can be seen, the value of the three images are relatively stable.

Table 1. Vertical and horizontal scanning results contrast

\begin{tabular}{|c|c|c|c|c|c|c|}
\hline \multirow{2}{*}{ Scanning times } & \multicolumn{2}{|c|}{ Image 1 } & \multicolumn{2}{c|}{ Image 2 } & \multicolumn{2}{c|}{ Image 3 } \\
\cline { 2 - 7 } & Horizontal & Vertical & Horizontal & Vertical & Horizontal & Vertical \\
\hline 1 & 138 & 136 & 193 & 193 & 153 & 152 \\
\hline 2 & 135 & 138 & 193 & 194 & 153 & 152 \\
\hline 3 & 140 & 136 & 193 & 192 & 152 & 153 \\
\hline 4 & 136 & 140 & 193 & 193 & 153 & 153 \\
\hline 5 & 139 & 139 & 192 & 194 & 154 & 152 \\
\hline 6 & 135 & 138 & 194 & 194 & 152 & 152 \\
\hline 7 & 139 & 139 & 194 & 194 & 153 & 152 \\
\hline
\end{tabular}

Refer to the search results of Table 1, respectively, use the SLIC and improved SLIC method to experiment.The results are shown in Figure 3 to 5, where in column (a) and column (c) ,the segmentation results call for manual placement of superpixel number ,and (b) are the improved SLIC segmentation results.

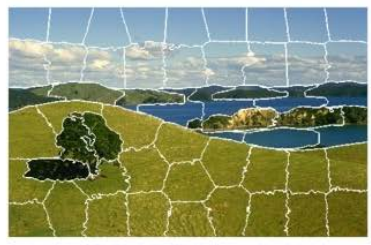

(a)superpixels $=60$

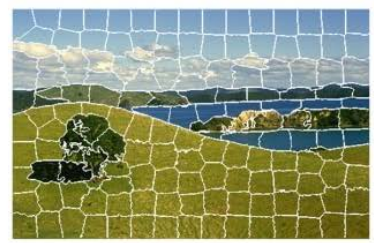

(b) superpixels $=138$

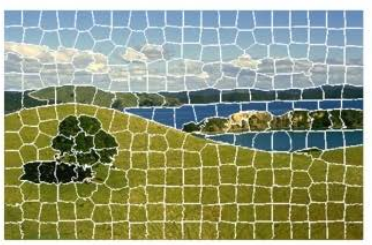

(c) superpixels $=300$

Figure 3. Image 1 segmentation results contrast 


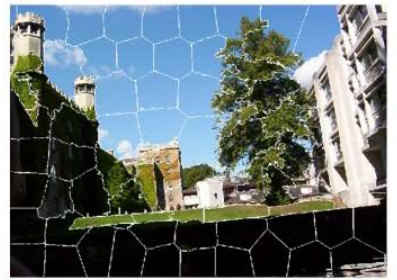

(a)superpixels $=80$

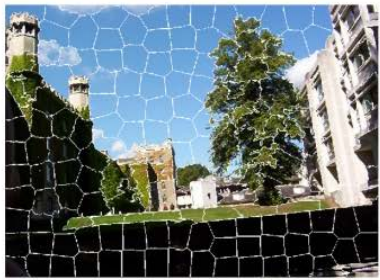

(b) superpixels $=193$

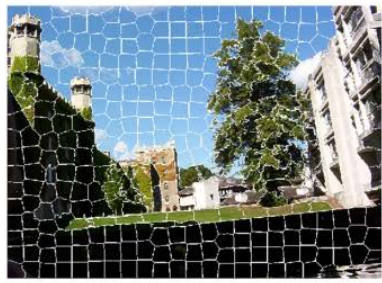

(c) superpixels $=400$

Figure 4. Image 2 segmentation results contrast

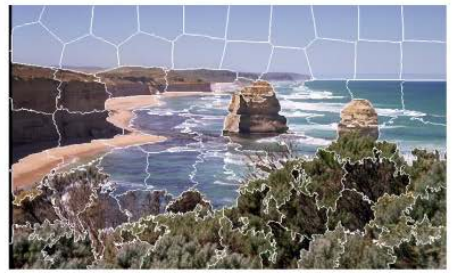

(a)superpixels $=80$

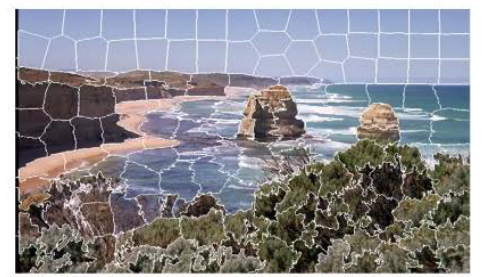

(b) superpixels $=153$

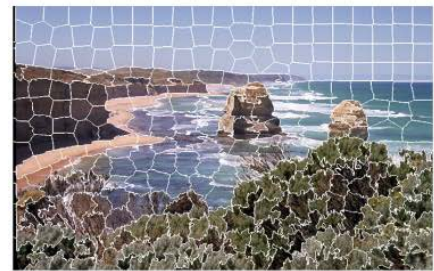

(c) superpixels $=300$

Figure 5. Image 3 segmentation results contrast

From Figure 3 (a), the distant mountain and lake are not completely divided, so as the sky and the clouds. The segmentation for the tree is not complete in Figure 4 (a) .For Figure 5 (a),the sand and the sea, the coast and the sky are splitted incomplete. For column (c) images, although the division are more accurate, but compared to the column (b) images,they are too close to the classify characteristics. Column (b) images not only do not over segment the imge,but also accurate segment each significant region image, verify the effectiveness of the improved SLIC method.

\section{Conclusion}

Combined with HSV non-uniform quantization,SLIC superpixel segmentation method not only avoids the trouble of artificial setting superpixel number, but also controls the over-segmentation and under-segmentation to $\mathrm{s}$ certain extent,so it meets the visual requirements of regional segmentation. The next work will be secondary classification of the image on the basis of the superpixel segmentation,thereby to complete the extraction work of salient regions.

\section{References}

[1]Ren X, Malik J. Learning a Classification Model for Segmentation[J]. Proc.int.conf.computer Vision, 2003, 1:10-17 vol.1.

[2]Shi J, Malik J. Normalized cuts and image segmentation[J]. IEEE Trans.pattern Anal.mach.intell, 2000, 22(8):888-905.

[3]Felzenszwalb P F, Huttenlocher D P. Efficient Graph-Based Image Segmentation[J]. International Journal of Computer Vision, 2004, 59(59):167-181.

[4] Moore A P, Prince S J D, Warrell J, et al. Superpixel lattices[C]. Computer Vision and Pattern Recognition, 2008:1-8.

[5]Comaniciu D, Meer P. Mean shift: a robust approach toward feature space analysis[J]. IEEE Transactions on Pattern Analysis \& Machine Intelligence, 2002, 24(5):603-619.

[6]Vedaldi A, Soatto S. Quick Shift and Kernel Methods for Mode Seeking[C].Computer Vision ECCV 2008. 2008:705-718.

[7] Achanta R, Shaji A, Smith K, et al. SLIC superpixels compared to state-of-the-art superpixel methods.[J]. IEEE Transactions on Pattern Analysis \& Machine Intelligence, 2012, 34(11):2274-82. 